\title{
Rosalind Thomas, Polis Histories, Collective Memories and the Greek World
}

Cambridge-New York, Cambridge University Press, 2019, pp. xii + 490, ISBN 9781107193581, £ 105.

\section{Sonny Wyburgh}

\section{(2) OpenEdition}

\section{Journals}

\section{Edizione digitale}

URL: https://journals.openedition.org/mythos/3505

DOI: $10.4000 /$ mythos.3505

ISSN: 2037-7746

Editore

Salvatore Sciascia Editore

\section{Notizia bibliografica digitale}

Sonny Wyburgh, «Rosalind Thomas, Polis Histories, Collective Memories and the Greek World », Mythos [Online], 15 | 2021, online dal 15 décembre 2021, consultato il 15 décembre 2021. URL: http:// journals.openedition.org/mythos/3505 ; DOI: https://doi.org/10.4000/mythos.3505

Questo documento è stato generato automaticamente il 15 décembre 2021.

Mythos 


\section{Rosalind Thomas, Polis Histories, Collective Memories and the Greek World}

Cambridge-New York, Cambridge University Press, 2019, pp. xii + 490, ISBN 9781107193581, £ 105.

\section{Sonny Wyburgh}

\section{NOTIZIA}

Rosalind Thomas, Polis Histories, Collective Memories and the Greek World, Cambridge-New York, Cambridge University Press, pp. xii + 490; ISBN 9781107193581; £105,00

La più recente monografia di Rosalind Thomas continua una linea di ricerca a cui la studiosa oxoniense aveva già dedicato due contributi pubblicati nel 2014 ('Local history, Polis history and the politics of place' in Parmeggiani (ed.), Between Thucydides and Polybius. The Golden Age of Greek Historiography, Washington, D.C., pp. 239-62; 'The Greek Polis and the Tradition of Polis History: local history, Chronicles and the Patterning of the Past' in Moreno - Thomas (edd.), Patterns of the Past. Epitedeumata in the Greek Tradition, Oxford, pp. 145-72). L'oggetto di studio è l'insieme di quelle opere storiografiche che l'Autrice denomina «local histories, better called polis and island

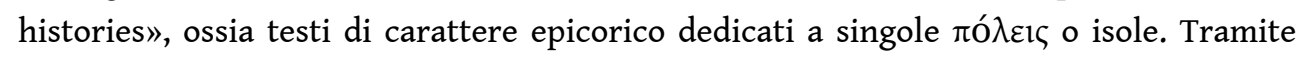
un'accurata ricostruzione storico-filologica di un corpus oggi frammentario, Thomas affronta il difficile compito di una contestualizzazione storico-culturale dei singoli casi di studio esaminati e del genere nel suo insieme. L'uso attento degli strumenti ermeneutici propri delle discipline antropologiche - tratto distintivo della produzione dell'Autrice sin da Oral Tradition and Written Record in Classical Athens (Cambridge-New York 1989) - e una scrupolosa attenzione alla storia della cultura offre una serie di considerazioni utili ad un'ampia gamma di studiosi della Grecia antica. Il volume analizza il modo in cui singole comunità del mondo greco potevano affermare la 
propria posizione nel più ampio panorama dell'Egeo attraverso la raccolta delle proprie memorie collettive, o meglio tradizioni, in testi scritti. Polis Histories ricostruisce e riqualifica un genere storiografico ingiustamente considerato secondario, ne discute le cause e le finalità in un'ottica socio-culturale e getta una luce sugli strumenti elaborati

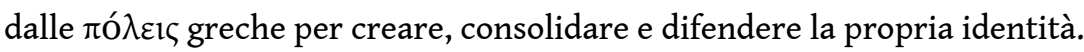

2 Il volume si compone di dieci capitoli seguiti da tre appendici analitiche, tra cui spicca Appendix 3: Register of Polis, Island and Ethnos Histories: Jacoby's Local Histories (pp. 417-45), uno strumento che integra le testimonianze raccolte nei volumi IIIA e B della raccolta di Jacoby con nuove scoperte, opere destinate al volume IV e opere inserite altrove dallo storico tedesco, sistemandole comodamente in ordine alfabetico per località. Grazie alla solida unità tematica e l'esauriente analisi dei casi di studio discussi, ciascuno di questi capitoli è fruibile autonomamente per chi fosse interessato ad approfondire singoli aspetti legati alla produzione storiografica presa in esame. L'aspetto più significativo di questo studio risiede tuttavia nell'insieme, ossia nella forza argomentativa con cui l'Autrice propone, testimonianza dopo testimonianza, di mettere in discussione la nostra percezione dello sviluppo di un pensiero storico nel mondo greco antico, ricostruendone una tappa non marginale. Come evidenzia sin dalle prime pagine della densa introduzione metodologica (pp. 1-28), «local historiography was the most common form of history-writing in these centuries [sc. IV-III]. [...] In the surge of research into the polis as an institution, remarkably little attention has been paid to the histories devoted to such political units. Nor do studies of historiography give much time to them» (p. 2). Nella stessa pagina, troviamo le tre domande analitiche che guidano la ricerca di Thomas. Perché i cittadini di un determinato luogo hanno sentito il bisogno di scrivere intere opere dedicate alla sua geografia, ai suoi culti e al suo passato? In che misura queste storie offrono un'immagine identitaria della propria

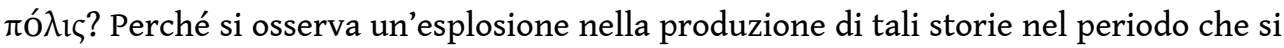
estende tra la fine del $\mathrm{V}$ e l'inizio del II secolo a.C.?

3 Il primo capitolo, What Are Polis Histories? What Are Local Histories? Popular Histories and Its Audiences (pp. 29-73), si pone l'obiettivo di delimitare il materiale da considerare, definirne la portata in relazione ai frammenti e le testimonianze in nostro possesso e delinearne l'orizzonte d'attesa. Il quadro che ne esce è estremamente ricco e

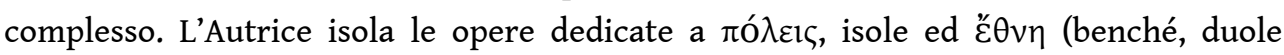
ammettere, a questa terza categoria dedicherà poi poco spazio) dell'area geografica che accerchia il mare Egeo prodotte prevalentemente in età ellenistica. Intrecciando un dialogo critico con i volumi IIIA, B e C di FGrHist, Thomas individua circa 530 autori che si sono occupati di questo genere di storiografia epicorica. Tale ricchezza di testimonianze consente a Thomas di stabilire che queste produzioni dovevano godere di una circolazione e fruizione che non si può spiegare soltanto tramite i gusti eruditi di una ristretta cerchia di poeti alessandrini. Si nota qui una delle vene polemiche dello studio: secondo l'Autrice, questo genere è stato vittima di un pregiudizio 'intellettualizzante' per una sua presunta natura 'antiquaria'. L'Autrice intende decostruire questa interpretazione, attribuendo un'utilità, se non un'esigenza, di natura socio-politica alla produzione e fruizione di tali storie. Ne derivano due ipotesi operative. La prima è che le grandi storie panelleniche di carattere rigidamente politico-militare rappresentate da Tucidide, Senofonte o Polibio fossero anticonvenzionali lungo tutto l'arco dell'epoca classica ed ellenistica. La seconda è che si 
debba inquadrare queste opere come «mass historiography» (p. 30), nel duplice senso di storiografia prodotta in massa e storiografia prodotta per la comunità.

4 Il secondo capitolo, Tales for the Telling: $\tau \dot{O} \mu \nu \theta \tilde{\omega} \delta \varepsilon \varsigma$ (pp. 74-99), sviluppa queste due conclusioni ricostruendo i contenuti e lo stile narrativo di queste storie. Partendo da alcuni passi di Polibio e di Dionigi d'Alicarnasso, l'Autrice stabilisce che questo genere godeva di una ampia divulgazione e si distingueva per un'attenzione marcata all'aspetto narrativo. In una temperie culturale che sta riscoprendo la validità pedagogica ed ermeneutica della nozione di «storytelling», non sorprende vederne l'uso da parte di Thomas per tradurre e discutere il concetto greco di tò $\mu v \theta \tilde{\omega} \delta \varepsilon \varsigma$ (p. 74). I lettori più esperti sanno che l'interesse dell'Autrice verso l'affabulazione nella trasmissione di tradizioni deriva dalle folte letture di studi etnografici e sociologici, come le sue bibliografie ben testimoniano. Attraverso l'analisi di due casi di studio (la «tale [sc. historic]» di Policrite a Nasso e quella di Enalo a Lesbo), l'Autrice mostra come queste opere contenessero racconti dotati di una propria potenziale autonomia semantica, rimanendo tuttavia intimamente connessi ad elementi identitari specifici. Erano storie che coinvolgevano diverse parti della comunità (non solo la classe dirigente maschile) e che toccavano le vicende di altri gruppi sociali e la fondazione di culti o la delimitazione di spazi.

Il terzo capitolo, Ethnography for the Greeks? The Polis as a New Subject for Historiography

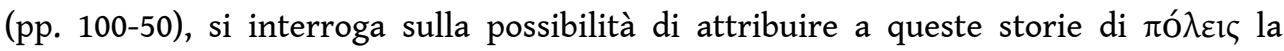
nozione moderna di etnografia. Attraverso l'analisi di alcuni esempi tratti dalle storie di Nasso, Paro e Delo, si osserva come rientrassero in queste opere excursus degni dei

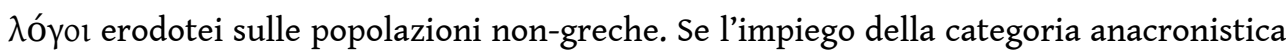
di 'etnografia' è ritenuto accettabile per Erodoto, l'attribuzione di tale etichetta agli scrittori di storie di đó $\lambda \varepsilon 1 \zeta$ suscita qualche difficoltà. In questo caso il punto di vista è interno alla stessa comunità e sembra mancare il rapporto egemonico subalterno tra osservatore e osservato costitutivo di una buona parte dell'etnografia del secolo scorso (doveroso il rimando a E. Said, Orientalism, New York 1979). La parte finale di questo capitolo offre un'utile discussione sugli strumenti ermeneutici disponibili agli studiosi di storiografica greca e sulla necessità di aggiornare le proprie categorie interpretative, sfumando le distinzioni fondate sullo stile e concentrandosi piuttosto sulle funzioni sociali di questi testi destinati alla auto-rappresentazione delle comunità greche.

6 Sulla marcatezza di una funzione pro-sociale del genere insiste il quarto capitolo, Fostering the Community: Accumulative Historiography (pp. 151-76), dedicato alle storie di Megara e Colofone. Lo stesso tema viene successivamente ripreso nel settimo capitolo, Polis in Flux: Dislocation and Disenfranchisement in Samos (pp. 275-315). Nel primo dei due, questo aspetto viene osservato nella produzione di opere storiografiche 'cumulative' se non propriamente 'corali'. La ripetizione degli stessi episodi in testi diacronicamente distinti suggerisce a Thomas di smontare il cliché dello storico recenziore che smentisce o corregge il predecessore. Secondo l'Autrice, la ripetizione avrebbe favorito la costituzione di un'identità politica (nel senso greco del termine) in un momento in cui essa era percepita come vacillante, fosse per la presenza di una comunità concorrente più influente (il caso di Megara) o per l'isolamento geografico e culturale dalle altre comunità elleniche (il caso di Colofone). Sono interessanti l'interpretazione dello storiografo come «embodiment of local memory or tradition» (p. 157) e il rapporto cognitivo tra ripetizione, scrittura e la nozione di verità: due punti che potrebbero essere ulteriormente svolti in un dialogo con gli studi sulla formularità nella poesia 
arcaica. Tramite un ricorso alla ripetizione dei contenuti, le storie di $\pi$ ó́ un'immagine identitaria unitaria del gruppo sociale. Nella stessa direzione, ma dalla prospettiva opposta, il capitolo settimo. Attraverso lo spoglio delle opere dedicate alla travagliata storia di Samo, si può constatare come una certa amnesia strutturale della comunità, attraverso una deliberata omissione dei dissidi politici interni, fosse funzionale a salvaguardare un'identità percepita come estremamente fragile. In entrambi i casi risulta efficace la conclusione di Thomas, secondo cui queste operazioni espressive servivano a "preserving or fostering the community» (p. 175).

Il quinto capitolo, Origins, Foundations and Ethnicity: Greeks and Non-Greeks (pp. 177-226), esplicita la tesi portante del volume: le storie di тó $\lambda \varepsilon ı \varsigma$ erano testi che raccoglievano le tradizioni della comunità per plasmarne un'immagine identitaria forte, in un contesto storico in cui tale identità era verosimilmente messa a repentaglio. Contribuendo al più ampio dibattito contemporaneo sulla esistenza o meno di una «ethnicity» ellenica, Thomas dedica questo capitolo alle storie di origini e fondazioni, che secondo l'Autrice dovevano costituire una porzione non indifferente di queste opere storiografiche. Attraverso lo studio dei casi offerti da Eritre, Mileto, Efeso e Chio, si dimostra come tali racconti - che molti studiosi stenterebbero a considerare 'storici' - rispecchino i dilemmi identitari e le tensioni diplomatiche delle comunità coinvolte. Più che uno strumento per ricostruire una storia arcaica delle $\pi$ ó $\varepsilon \varepsilon ı \zeta$ ioniche, questi racconti sono una lente d'ingrandimento privilegiata su come ciascuna comunità rendeva conto della sua vicinanza ad un mondo non-ellenico e dell'assenza dai grandi cicli leggendari dell'epoca eroica. Le conclusioni che l'Autrice trae, su una eroicizzazione della colonizzazione ionica e l'inserimento di «pre-origins» cretesi per rivendicare una 'grecità' antica e indipendente da Atene, offrono spunti utilissimi per chiunque si appresti a studiare il patrimonio leggendario ellenico. Solo attraverso una corretta storicizzazione di questi racconti è possibile leggerli in maniera non esclusivamente letteraria.

8 Questo interesse costante per una corretta storicizzazione dei contenuti delle storie di

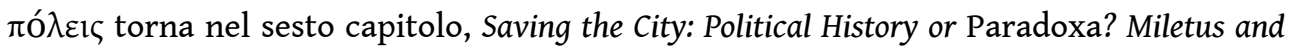
Lesbos (pp. 227-74). Attraverso l'analisi di opere dedicate a Mileto e a Lesbo, l'Autrice mostra come all'interno dello stesso genere potessero coesistere scelte contenutistiche totalmente divergenti. Le storie su Mileto tendono a privilegiare l'aspetto politico ed istituzionale della comunità, mentre i racconti su Lesbo - che aveva pur avuto una vivace attività politica, come la poesia arcaica testimonia - si concentrano su elementi cultuali o mitologici. Ricostruendo il diverso statuto delle due città nel IV secolo, Thomas dimostra come la scelta dei contenuti dovesse essere strettamente legata a contingenze storico-sociali. L'Autrice sostiene, in modo convincente, che in entrambi i casi si possa parlare di iotopíal, suggerendo dunque che l'unità di genere sia da intravedere nelle finalità delle opere piuttosto che nei loro contenuti.

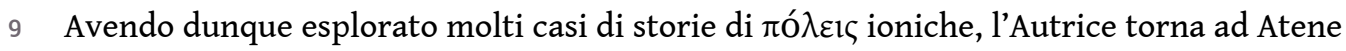
nei due capitoli che precedono le conclusioni. In Athenian Polis Histories (pp. 316-57), si evince quanto sia facile interpretare erroneamente le storie epicoriche dell'Attica se non le si inquadra nel contesto storico-culturale in cui sono nate. Come l'Autrice ha

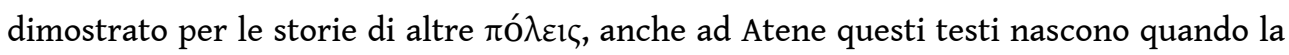
comunità inizia a percepire un decadimento del proprio peso politico e della propria identità sullo scacchiere panellenico. La scrittura di queste storie è dunque «a form of defence and self-preservation» (p. 340). Nel penultimo capitolo, The Aristotelian Politeiai 
and Local History (pp. 358-85), l'Autrice mostra come un genere spesso considerato ancillare allo studio del pensiero politico di Aristotele sia in realtà molto simile alle

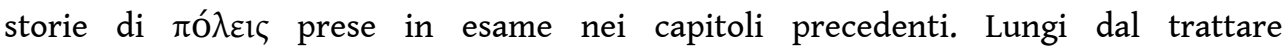
esclusivamente le 'costituzioni' in senso moderno, questi testi descrivono storia locale, usi e geografia di una comunità, fornendo racconti tradizionali di tipo leggendario ed eziologie per luoghi significativi o istituzioni dotate di valore identitario. Ciò che li distingue, come la stessa Thomas precisa, è il fatto di essere redatti da persone estranee alle $\pi 0 ́ \lambda \varepsilon 1 \zeta$ e di presentarsi come un progetto sistematico di ricerca volto a documentare e preservare gli aspetti costitutivi della ró $\lambda_{1} \zeta$ come entità sociale. Il risultato, nelle parole dell'Autrice, fu quello di aver creato "stylized visions of community, an 'imagined community' with customs, political institutions, origins and origin myths and some (or more) of its past history» (p. 385).

10 L'attenta ricostruzione di contenuti, stile, produzione e funzione delle storie di $\pi$ ó $\lambda \varepsilon 1 \varsigma$ permette a Thomas, nel capitolo conclusivo Polis and Island Histories and the Late Classical and Hellenistic World: A New Hellenism? (pp. 386-409), di rispondere alle domande con cui il volume si apre. Sottolineando l'importanza della categorizzazione moderna delle fonti e delle testimonianze ai fini della comprensione dei realia, l'Autrice lancia un appello a studiare ulteriormente, aggiungerei seguendo il percorso da lei inaugurato, il modo in cui le diverse comunità greche scrivevano della propria ró $\lambda_{\imath \varsigma}$. Come suggerisce il titolo del capitolo, un'indagine di questo genere potrebbe consentire alla comunità scientifica di attribuire al periodo successivo alla guerra del Peloponneso lo sviluppo di un 'ellenismo' non più basato sulla coppia oppositiva "Greci - Barbari”, ma orientato piuttosto verso una multi-direzionalità interna, da intendere come «a celebration of differences within the shared culture» (p. 397).

11 Si comprende dunque perché il volume abbia ricevuto il titolo di Polis Histories, Collective Memories and the Greek World. Oltre a presentarsi come strumento fondamentale per qualsiasi successivo studio sullo sviluppo della storiografia in Grecia antica, il lavoro di Thomas offre una nuova prospettiva, sanamente critica e problematica, sulla nostra rappresentazione mentale di un 'mondo' greco. Tale elaborazione non può prescindere dallo studio attento delle immagini e dalle narrazioni con cui le comunità che componevano tale 'mondo' si auto-rappresentavano, un precetto metodologico che questo studio contribuisce a corroborare. Con le parole che ne concludono le oltre 400 pagine, l'Autrice lascia i lettori con una riflessione che, parlando degli scrittori di storie di $\pi$ ó $\lambda \varepsilon ı \zeta$, si addice bene come indicazione agli storici della Grecia antica di oggi: «Historiography served this purpose. It stressed the diversity of polities at the same time as emphasizing a binding set of ties that served to sustain a Hellenic unity amidst the multiplicity of poleis and polities» (p. 409). 


\section{AUTORI}

SONNY WYBURGH

Università degli Studi di Pisa,

sonny.wyburgh(at)phd.unipi.it 